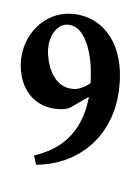

\title{
UNA EXPERIENCIA EN LA VINCULACIÓN UNIVERSIDAD-EMPRESA: EL PROYECTO COGEMPLEO DE LA FUNDACIÓN CAMPUS TECNOLÓGICO DE ALGECIRAS
}

(AN EXPERIENCE IN UNIVERSITY-ENTERPRISE RELATIONS:

THE COGEMPLEO PROJECT IN THE TECHNOLOGICAL FOUNDATION CAMPUS OF ALGECIRAS)

\author{
María José Foncubierta Rodríguez \\ Universidad de Cádiz. \\ José Luis Perea Vicente \\ Consultoría de Finanzas, Cádiz \\ Gabriel González Siles \\ Universidad de Cádiz
}

DOI: 10.5944/educXX1.15584

\section{Cómo referenciar este artículo/How to reference this article:}

Foncubierta Rodríguez, M. J.; Perea Vicente, J. L. y González Siles, G. (2016). Una experiencia en la vinculación Universidad-Empresa: El proyecto cogempleo de la fundación campus tecnológico de Algeciras. Educación XX1, 19(1), 201-225, doi:10.5944/educXX1.15584

Foncubierta Rodríguez, M. J.; Perea Vicente, J. L. \& González Siles, G. (2016). Una experiencia en la vinculación Universidad-Empresa: El proyecto cogempleo de la fundación campus tecnológico de Algeciras. [An experience in university-enterprise relations: the cogempleo project in the technological foundation campus of algeciras]. Educación XX1, 19(1), 201-225, doi:10.5944/ educXX1.15584

\section{RESUMEN}

La puesta en marcha del denominado Espacio Europeo de Educación Superior ha traído consigo un mayor acercamiento entre la enseñanza superior y el mundo laboral. No obstante, aún son escasos los estudios que tengan como base encuestas dirigidas al entorno empresarial sobre el ajuste del perfil de competencias adquiridas por el titulado en relación con las necesidades demandadas por aquel.

En este trabajo analizamos un proyecto llevado a cabo en la comarca del Campo de Gibraltar por parte de la Fundación Campus Tecnológico de Algeciras, denominado Proyecto COGEMPLEO. En el mismo, la Fundación ha puesto en contacto al mundo académico con el tejido empresarial de la zona, a través de comités de representantes de los colectivos de profesorado, alumnado y egresados de las titulaciones de la Escuela Politécnica Superior 
de Algeciras (EPSA), de la Universidad de Cádiz, y del entorno empresarial comarcal: PYMES, grandes empresas e instituciones empresariales.

Utilizando el método Delphi, se obtuvo un Modelo de trabajo en Competencias Genéricas, y, derivado de él, un Perfil mínimo deseado del egresado en dichas competencias. A partir del mismo, se diseñaron un conjunto de acciones, factibles y efectivas, que acercan el perfil real del estudiante al consensuado por las partes. Asimismo, los colectivos reconocen el valor de este proyecto como modelo de trabajo para dar respuesta a sus necesidades de cooperación, con la finalidad última de otorgar empleabilidad a la población activa del entorno.

\section{PALABRAS CLAVE}

Empleabilidad; Competencias; Universidad; Transición al mercado laboral; Empleadores; Entorno empresarial.

\section{ABSTRACT}

The European Higher Education Area (EHEA) has brought about an approach between the University and the labour market. However, there are not many investigations based on enquiries to the business network about the adjustment of the competencies profile acquired by the graduate in relation to the business network needs demanded.

In this paper we analyze a project carried out in the zone of Campo de Gibraltar by the Algeciras Technological Foundation Campus, called the COGEMPLEO Project. In this project, the Foundation has linked the academic world with the business network of the zone, through representative committees of professors, students and EPSA graduates of the University of Cádiz, and the business network through SMES, big companies and business institutions.

Using the Delphi Method, they obtained a Generic Competences Model, and from the same, they obtained the minimum desired graduate profile in these competences. Based on that, a group of actions, both feasible and effective, was designed to approach the true profile of the student to this profile. Furthermore, the parties recognize the value of this project as a model of work to provide an answer to the cooperation requirements with the ultimate purpose of providing employability for the zone's labor force.

\section{KEY WORDS}

Employment; Job Skills; Higher Education; Employers; Social Network. 
MARÍA JOSÉ FONCUBIERTA RODRÍGUEZ, JOSÉ LUIS PEREA VICENTE Y GABRIEL GONZÁLEZ SILES

\section{COMPETENCIAS GENÉRICAS EN LA EDUCACIÓN SUPERIOR: LA EMPLEABILIDAD COMO OBJETIVO}

La idea europea de crear un área de educación superior integrada (el Espacio Europeo de Educación Superior (EEES), derivado de la Declaración de Bolonia de 1999) para agilizar la movilidad de los recursos humanos cualificados entre sus países, concluyó, entre otras acciones, en el Proyecto Tuning, Tuning Educational Structures in Europe (González y Wagenaar, 2003). Según el informe Education at a glance (OCDE, 2008) «las economías de los países integrados en la OCDE dependen cada vez más de que exista una oferta estable de trabajadores bien preparados» (Martín, Rabadán y Hernández, 2013, p. 246). Para ello, la movilidad de estudiantes y egresados en territorio europeo requería, ante todo, información fiable y objetiva (principios de transparencia, cooperación y confianza mutua) sobre los programas educativos, sobre una estructura comparable de los curriculum vitae, y, de cara al mercado laboral, sobre qué competencias supone un título concreto y qué significan estas en la práctica.

El Proyecto Tuning introdujo el concepto de «resultados del aprendizaje», definiéndolos como el conjunto de competencias que incluye conocimientos, comprensión y habilidades, que se espera que el estudiante domine, comprenda y demuestre después de completar un proceso corto o largo de aprendizaje. Dichas competencias pueden ser genéricas o específicas de cada área temática y resultan sumamente relevantes, por tanto, para la mejora de la empleabilidad, la ciudadanía activa y el desarrollo personal en el aprendizaje permanente. Este proyecto se ha basado en tres pilares informativos: el colectivo académico, el de egresados y el de empleadores ${ }^{1}$. Es una confirmación de la búsqueda de la empleabilidad en la formación de los estudiantes.

Van der Hofstadt y Gómez (2006) indican que el estudiante universitario ha de aprender en primer lugar aquellas competencias genéricas que le asistan a conseguir y mantener un empleo, como actitud activa en la búsqueda de trabajo, habilidades de comunicación y otras para afrontar una entrevista, o el desarrollo de un proyecto empresarial propio, como alternativa laboral. Se ha demostrado que la velocidad con la que los graduados aprenden a desarrollar su trabajo depende del nivel y del tipo de competencias adquiridas en la educación formal (Heijke, Meng y Ris, 2003). No obstante, Calderón y Escalera (2008) señalan que con la puesta en marcha del EEES, el reto de la universidad es impulsar el aprendizaje activo y comprometido del estudiante, organizando su educación alrededor de dichas competencias, pero, propiciando el desarrollo del pensamiento crítico que es, frente a otras enseñanzas superiores enfocadas a la profesionalización, el gran valor añadido y el auténtico sello de identidad de la enseñanza universitaria. 
Admitiendo, según los estudios mencionados, que las competencias genéricas hacen al egresado «más empleable», la cuestión que surge es: ¿qué competencias principalmente? En este sentido, lo primero es definir qué se entiende por competencia genérica.

La Real Academia de la Lengua Española define competencia como pericia, aptitud, idoneidad para hacer algo o intervenir en un asunto determinado. Jornet, González, Suárez y Perales (2011, p. 125) señalan que son competencias «todos aquellos aspectos (habilidades, destrezas, actitudes...) que debe poseer un profesional para desarrollar su labor de una forma eficaz». Ribes (2011, p. 36) sostiene que «ser competente implica siempre un hacer determinado, un dominio técnico, una pertinencia o incumbencia práctica respecto de algo que se traduce en logros y resultados. Ser competente no tiene nada que ver con estar informado, con ser erudito o estar especializado. Ser competente siempre se refiere a una forma de intervención o hacer específicos en dominios específicos». Villa y Poblete (2011, p. 148) lo definen como «la integración de una serie de elementos (conocimientos, técnicas, actitudes, procedimientos, valores) que una persona pone en juego en una situación problemática concreta demostrando que es capaz de resolverla». Por otra parte, varias son las expresiones utilizadas para designar el concepto de competencias genéricas: competencias transferibles, competencias clave, competencias básicas, competencias centrales, competencias personales y competencias para el empleo, aunque todas ellas pueden ser consideradas sinónimas (Fallows y Steven, 2000).

Las competencias de naturaleza genérica cualifican a las personas en capacidades transversales (metodológicas, personales y sociales), útiles en muy diversas funciones, tareas y ámbitos. Garantizan el desarrollo de una verdadera educación integral para ser ciudadanos activos y profesionales competentes (Blanco, 2009). Desde el punto de vista profesional, ser competente consistiría en «saber sobre (conocimientos); saber cómo intervenir (conjunto de habilidades y destrezas cognitivas, emocionales, sociales y procedimentales que permiten aplicar el conocimiento que se posee); saber relacionarse (disponer de habilidades sociolaborales); saber utilizar estratégicamente el conocimiento y perfeccionarse (disponer de competencias metacognitivas); y saber comportarse (ajuste a valores, principios, creencias y actitudes profesionalmente válidas y colectivamente aceptadas)» (Vélaz de Medrano, 2008, p. 158).

En el mismo sentido, Corominas $(2001,2006)$ señala que las competencias genéricas son aquellas de tipo cognitivo, sociales, emocionales y éticas que tienen carácter transferible y que han de suponer el «saber ser» y el «saber estar» de la educación superior, mientras que las competencias específicas se refieren al saber hacer y al saber guiar. Dominar ambos tipos 
de competencias capacitan al individuo para desarrollar eficientemente una labor profesional (Tejada y Navío, 2005). Estos autores, no obstante, añaden una condición relevante al indicar que las competencias genéricas suponen realmente una ventaja competitiva para la empresa y un factor de empleabilidad para la persona solo y cuando la entidad se gestione y se estructure organizativamente mediante un modelo de competencias, es decir, lleve a cabo una verdadera "organización por competencias». Cuestión que irán aplicando las empresas progresivamente, al menos en lo relativo al análisis y valoración de puestos y funciones, en opinión de Gutiérrez y De Pablos (2010), pues en la actualidad los puestos de trabajo han pasado, por lo general, a ser complejos y a conllevar mayores responsabilidades y múltiples funciones, con lo que requieren competencias de adaptación, iniciativa, asunción de responsabilidad, etc.

En España, la Ley Orgánica 4/2007 de Universidades, de 12 de abril, por la que se modifica la Ley Orgánica 6/2001, de 21 de diciembre, establece una nueva estructura de las enseñanzas y títulos universitarios españoles en consonancia con los objetivos establecidos para la construcción del EEES. En el Real Decreto 1393/2007, por el que se determina la ordenación de las enseñanzas oficiales universitarias (BOE n. ${ }^{\circ} 260$, de 30 de marzo de 2007) se expone que los planes de estudios conducentes a la obtención de un título deberán, por tanto, tener en el centro de sus objetivos la adquisición de competencias por parte de los estudiantes, ampliando, sin excluir, el tradicional enfoque basado en contenidos y horas lectivas. En su Anexo I «Memoria para la solicitud de verificación de Títulos Oficiales», el punto 3.2, dentro de Objetivos de los títulos, se relacionan las competencias básicas que, como mínimo, deberán adquirir los estudiantes de un título de Grado.

Complementario al trabajo del Proyecto Tuning, un grupo internacional de expertos, agrupados bajo el nombre de Joint Quality Initiative (JQI) estableció los denominados Descriptores de Dublín (US, 2003), que enuncian genéricamente las expectativas típicas respecto a los logros y habilidades relacionados con las cualificaciones que representan el fin de cada ciclo universitario del EEES (Grado, Máster y Doctorado). Esto es, se refieren a las competencias genéricas (en el nivel correspondiente a cada ciclo) que un estudiante debe ir desarrollando durante su proceso formativo, las cuales son:

- Poseer y comprender conocimientos en un área de estudio.

- Aplicar conocimientos a su trabajo o vocación de una forma profesional.

- Elaborar y defender argumentos.

- Resolver problemas dentro de su área de estudio. 
- Reunir e interpretar datos relevantes.

- Emitir juicios críticos y establecer conclusiones basados en los datos, que indiquen una reflexión personal sobre temas relevantes.

- Transmitir información, ideas, problemas, soluciones a público de distinto nivel.

- Desarrollar autonomía en el aprendizaje.

La Agencia Nacional Española para la Evaluación de la Calidad (ANECA), por su parte, recoge estas competencias cuando señala los requisitos para la verificación de los nuevos títulos (programa VERIFICA).

En definitiva, la norma española obliga a incluir y evaluar la adquisición de competencias genéricas en los títulos de Educación Superior, aunque esta no siempre resulta una tarea fácil. Al respecto, el Informe «Del diseño a la evaluación de las competencias genéricas: análisis empíricos e intervención mediante rúbricas» del Instituto Universitario de Educación a Distancia, de la Universidad Nacional de Educación a Distancia (IUED, 2010) concluye en su estudio que en un alto porcentaje, el 67,7\% del total de los títulos universitarios de Grado de la muestra de su investigación, que fue de 74 universidades españolas, no se hace alusión alguna a la evaluación de las competencias genéricas. Este dato, sin embargo, contrasta con el hecho de que el 76,93\% de las guías de las asignaturas sí recogen competencias genéricas entre los objetivos de dichas asignaturas. De ello deducen que es la fase de evaluación de dichas competencias la que plantea aún dificultades.

\section{EL PROYECTO COGEMPLEO DE LA FUNDACIÓN CAMPUS TECNOLÓGICO DE ALGECIRAS}

\section{Fundamentación, antecedentes y objetivos}

\section{Fundamentación}

Este proyecto es una iniciativa de la Fundación Campus Tecnológico de Algeciras (FCTA), institución sin ánimo de lucro, promovida por las Consejerías de Innovación, Ciencia y Empresa, y de Educación y Empleo de la Junta de Andalucía, la Universidad de Cádiz y el Ayuntamiento de Algeciras, constituida el 25 de septiembre de 2007. Su finalidad principal es la de responder a las necesidades de la sociedad campogibraltareña y contribuir al desarrollo económico de la Bahía de Algeciras ${ }^{1}$, a través de un marco innovador que engloba el conjunto de formación, capacitación, investigación e innovación vinculados a la sociedad del conocimiento, mediante una 
estructura única que coordina la formación profesional, la formación continua y ocupacional (formación para el empleo), la Educación Superior y los centros I+D+I de la Bahía de Algeciras y su entorno. Concretamente, en sus Estatutos Fundacionales se expone que entre sus objetivos está el de dar respuesta a la demanda formativa del mercado laboral, en los distintos niveles, en relación a la realidad tecnológica y empresarial del entorno de la Bahía de Algeciras.

A tal fin, y en el marco concreto de uno de los principales focos de actuación de la relación Fundación-Empresas, el complejo industrial, petroquímico, metalúrgico, energético y portuario, característico de la comarca del Campo de Gibraltar (considerado primer polo industrial de Andalucía y segundo de España), esta entidad se propuso iniciar un conjunto integral de actuaciones que acercase el perfil profesional de los estudiantes de las titulaciones relacionadas con las Ingenierías en Tecnologías Industriales que se imparten en el Campus Universitario de la Bahía de Algeciras, de la Universidad de Cádiz (UCA), a las demandas verdaderas de dichas empresas. Es en este contexto en el que surge el Proyecto COGEMPLEO: Competencias genéricas para el empleo. Su objetivo es definir un Modelo de Competencias Genéricas y un Perfil de Competencias Genéricas a desarrollar entre los estudiantes de la UCA en el Campo de Gibraltar, con objeto de mejorar su empleabilidad y su capacidad de acceso al mundo laboral.

\section{Antecedentes}

Son diversos los estudios relativos a la implementación de las competencias genéricas en la enseñanza superior. Podemos mencionar proyectos a nivel europeo e internacional (Tuning, CHEERS, REFLEX ${ }^{2}$, UEConverge ${ }^{3}$, proyecto $\mathrm{DeSeCo}^{4}$, etc.), en el marco de las propias universidades, nacionales e internacionales (Universidad Politécnica de Madrid, Universidad Autónoma de Barcelona, Universidad de Deusto, UNED, Universidad de Oviedo, Universidad de Alcalá de Henares, Universidad de Huelva, Universidad Cambridge ${ }^{5}$, proyecto TRANSEND-Universidad de Surrey ${ }^{6}$, etc.), desde la esfera empresarial (modelo de competencias genéricas para los mandos directivos e intermedios, cualificaciones profesionales para la gestión de los recursos humanos en una empresa, etc.), así como otras propuestas y categorizaciones que, derivadas de estos estudios y otros, se han realizado sobre dichas competencias (modelo genérico de competencias conductuales de Ansorena, modelo de la Agencia de Calidad Catalana, AQU, relación de competencias propuestas por Bennett, Dunne y Carre, de 1999, etc.).

Todos ellos coinciden en señalar la relevancia de las competencias genéricas en el desarrollo profesional y personal del individuo. Por lo general, la 
iniciativa ha partido desde el ámbito académico, dentro de las Universidades, o incluso político (proyectos europeos, DeSeCo). Está presente en todos ellos la empleabilidad como uno de los fines principales, y, por tanto, las necesidades del entorno económico-empresarial. No obstante, el caso que describimos en este artículo tiene un origen algo peculiar, pues comienza a gestarse como respuesta de la FTCA a las demandas que el marco empresarial de la gran industria de la Bahía de Algeciras venía haciendo con respecto a este tipo de competencias. La necesidad existía, y era manifiesta, la FTCA la recoge y trabaja en definir exactamente qué tipo de competencias reclaman las empresas, y, dentro de esa tipología, cuáles en concreto son las más demandadas.

Para el planteamiento del Proyecto COGEMPLEO se han tenido en cuenta, concretamente, los trabajos, y con el propósito, reflejados en la tabla 1.

\section{Tabla 1}

Estudios que han servido de referentes en la fase inicial del Proyecto COGEMPLEO

\begin{tabular}{|c|c|}
\hline $\begin{array}{l}\text { PROYECTO, MODELO O ESTUDIO } \\
\end{array}$ & FINALIDAD \\
\hline Proyecto Tuning & \multirow{2}{*}{$\begin{array}{l}\text { Definición y tipologia de las } \\
\text { competencias genéricas }\end{array}$} \\
\hline Modelo Genérico de Competencias Conductuales de Ansorena. & \\
\hline Modelo de Competencias Genénicas, Universidad de Deusto & \multirow{5}{*}{$\begin{array}{l}\text { Visión del ámbito } \\
\text { académico de enseñanza } \\
\text { superior }\end{array}$} \\
\hline Modelo de Competencias Genéricas, UNED & \\
\hline $\begin{array}{l}\text { - Análisis sobre aplicaciones de los Modelos de Competencias } \\
\text { Genéricas a la Educación Superior de la Universidad Autónoma de } \\
\text { Barcelona y de la Universidad Politécnica de Madríd }\end{array}$ & \\
\hline $\begin{array}{l}\text { Análisis de Competencias para la titulación de Ingeniero } \\
\text { Informático, Universidad de Oviedo }\end{array}$ & \\
\hline $\begin{array}{l}\text { Modelo de Competencias Genéricas en el Grado de Ciencias } \\
\text { Ambientales en España, coordinado por la Universidad de Alcalá. }\end{array}$ & \\
\hline $\begin{array}{l}\text { - Aplicaciones del modelo de Competencias Genéricas a diversas } \\
\text { áreas y asignaturas en la UCA (Organización del Trabajo, } \\
\text { Administración y Organización de Empresas, Competitividad e } \\
\text { Innovación, Contabilidad,...) }\end{array}$ & \multirow{2}{*}{$\begin{array}{l}\text { Visión académica, } \\
\text { primeras experiencias en } \\
\text { la Universidad de Cádiz, } \\
\text { y en su Campus } \\
\text { Universitario de la Bahía } \\
\text { de Algeciras }\end{array}$} \\
\hline $\begin{array}{l}\text { - Aplicaciones de desarrollo de Competencias Genéricas en títulos de } \\
\text { Postgrado de la UCA en el Campo de Gibraltar }\end{array}$ & \\
\hline Proyecto DiezC de la Cátedra CEPSA y la Universidad de Huelva & $\begin{array}{l}\text { Visión académica. } \\
\text { Proyecto con similar } \\
\text { punto de partida: la gran } \\
\text { empresa del entomo local }\end{array}$ \\
\hline $\begin{array}{l}\text { - Estudio de Competencias Conductuales para Mandos Intermedios y } \\
\text { Técnicos en una empresa industrial de ámbito nacional }\end{array}$ & \multirow{3}{*}{ Visión empresarial } \\
\hline $\begin{array}{l}\text { - Modelo de Cualidades Profesionales en una empresa de ámbito } \\
\text { nacional }\end{array}$ & \\
\hline $\begin{array}{l}\text { - Modelos generales de Competencias Genéricas para la gestión } \\
\text { empresarial }\end{array}$ & \\
\hline
\end{tabular}

Fuente: Elaboración propia a partir del documento interno de la FCTA: COGEMPLEO: Competencias Genéricas para el Empleo. Proyecto de Desarrollo de Competencias Genéricas para estudiantes de la Universidad de Cádiz (2009). 
Como mencionamos en la tabla anterior, en el Campus Universitario de la Bahía de Algeciras ya se habían realizado experiencias al respecto, con la petición, por parte de la Cátedra CEPSA de la UCA de incluir en la formación de los tres postgrados que la Escuela Politécnica Superior de Algeciras estaba impartiendo, y donde la citada cátedra era patrocinadora, determinadas competencias genéricas. Las titulaciones, Experto Universitario en Mantenimiento, Experto Universitario en Refino del Petróleo y Experto Universitario en Seguridad Industrial, estaban dirigidos a ingenieros y técnicos. El trabajo se realizó durante el curso 2006/07, y para seleccionar dichas competencias se tuvo en cuenta el Modelo de Competencias genéricas que utilizaba el Departamento de Desarrollo del Grupo CEPSA. Esta misma Cátedra tuvo un peso fundamental a la hora de plantear a la Fundación un estudio más amplio, que recogiese las necesidades sobre dichas competencias del entorno económico-empresarial, con la finalidad de aplicar, no ya solo a unas titulaciones concretas, sino a un conjunto de actuaciones a medio y largo plazo que concienciasen y cualificasen en las mismas a los estudiantes de la Bahía.

En este sentido, el Proyecto COGEMPLEO tiene una génesis similar al «DiezC» de la Universidad de Huelva, en la que, en el curso 2009/10, y de la mano de la Cátedra CEPSA, la Universidad de Huelva puso en marcha un modelo de actuación que pretende incentivar y ofrecer formación en competencias genéricas a su alumnado así como reconocer y premiar, con el certificado «Alumn@ DiezC», a los mejores alumnos por su desarrollo en este tipo de competencias. Unos años antes, en el curso 2005/06, la Fundación General de la Universidad de León y de la Empresa (FGULEM) había desarrollado, conjuntamente con el portal UNIVERSIA, patrocinado por el Grupo Santander, el modelo CERTICAP-UNIVERSIA ${ }^{7}$. Se trata de un sistema de evaluación de las competencias mediante una herramienta automatizada, reconocida a nivel internacional, lo que facilita el proceso de selección de personal para una empresa.

\section{Objetivos}

Tras los precedentes mencionados, y en la filosofía de orientar a los estudiantes a la adquisición de las competencias genéricas que se determinen como las más importantes para su perfil profesional, la Fundación define los siguientes objetivos para el Proyecto COGEMPLEO:

- Poner a disposición del estudiante medios para ampliar y perfeccionar las capacidades requeridas en el mercado laboral,

- Facilitar a las empresas egresados con una formación integral y unos niveles de competencias tanto técnicas como conductuales, acordes a los requisitos de los empleos, 
y, consecuentemente:

- Identificar el perfil de competencias genéricas a potenciar, de acuerdo a las necesidades del entorno empresarial y diseñar un programa de desarrollo en competencias genéricas para estudiantes (gráfico 1).

- Aplicar esta información en los servicios de orientación laboral y bolsa de trabajo que la propia Fundación está diseñando para el alumnado del Campus Universitario de la Bahía de Algeciras.

Gráfico 1. Relación Universidad/Fundación Campus Tecnológico y Empresas: objetivo del Proyecto COGEMPLEO

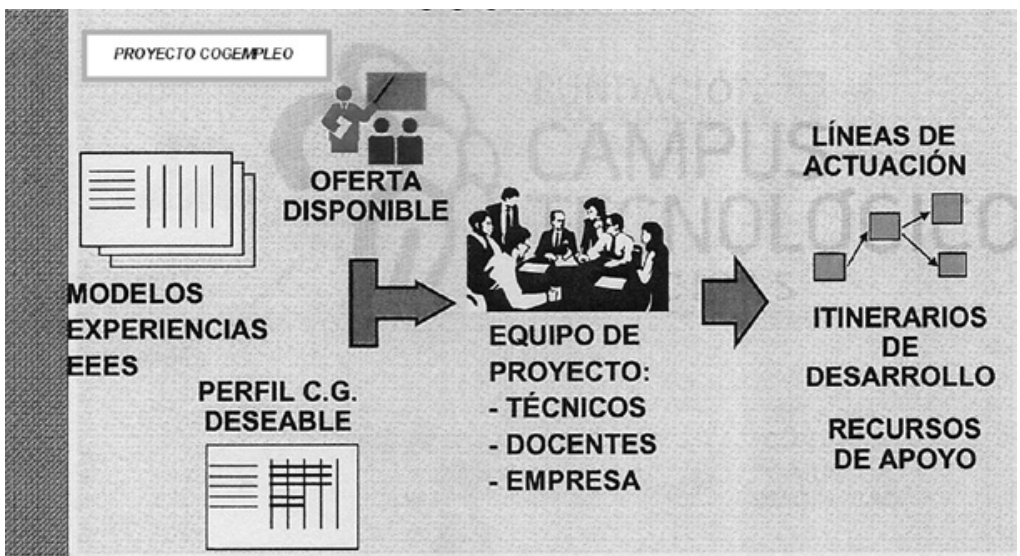

Fuente: FCTA. Presentación del Proyecto COGEMPLEO a los agentes sociales de la comarca del Campo de Gibraltar (julio, 2009)

El proceso reflejado en el gráfico anterior se caracteriza por ser participativo (contar con los colectivos sociales implicados: empresas, entidades y agentes sociales), de consenso, realista (con la finalidad de planificar actuaciones con garantía de éxito), de apoyo al desarrollo del estudiante, y abordable, pues está centrado en estudiantes y egresados de la EPSA.

\section{Proceso de investigación: metodología, desarrollo y resultados}

\section{Metodología}

Dado el carácter prospectivo del estudio, y la conveniencia de conocer las opiniones de un numeroso grupo de expertos, dentro de los cuatro colectivos: grandes empresas, PYME y empresas auxiliares, profesorado y egresados, se decidió utilizar el método Delphi. El trabajo investigador fue realizado entre junio de 2009 y julio de 2010. 
MARÍA JOSÉ FONCUBIERTA RODRÍGUEZ, JOSÉ LUIS PEREA VICENTE Y GABRIEL GONZÁLEZ SILES

Las fases del proceso quedan esquematizadas en el gráfico 2 .

Gráfico 2. Fases del método Delphi llevado a cabo en el Proyecto COGEMPLEO

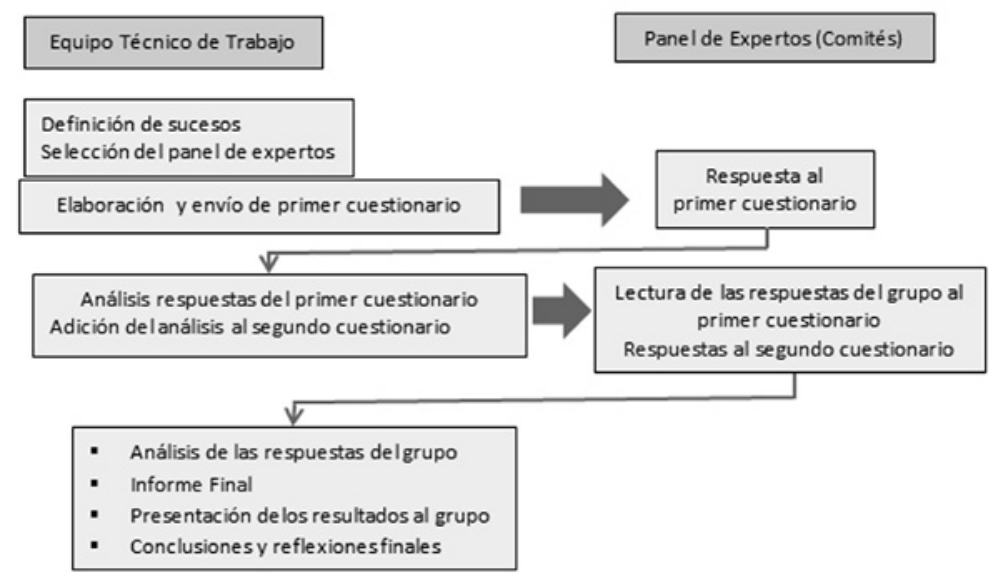

Fuente: Elaboración propia a partir de Moreno (2002) y Mengual (2011).

El equipo técnico de trabajo integrado por el director del Departamento de Desarrollo de la Refinería «Gibraltar» de CEPSA, el Gerente de la FCTA, un profesor titular de Ingeniería Industrial de la EPSA, con amplia experiencia en cargos académicos, y la Técnica de Formación de la FCTA.

\section{Desarrollo}

1. ${ }^{a} \quad$ Fase: análisis de modelos, definición de competencias y determinación del panel de expertos

Análisis de modelos y definición de competencias

El equipo técnico procedió a realizar una comparativa entre los modelos indicados en los antecedentes, equiparando terminologías y denominaciones de competencias entre ellos para posibilitar su comparación. Se detectaron las competencias que más se repetían entre estos. Asimismo, en aquellos casos en que se disponía de información, se tuvieron en consideración, como especialmente significativas, las opiniones de los representantes empresariales, por el propio objetivo final del proyecto. Se buscaba un modelo único de referencia para el estudio, así como una primera propuesta de Perfil a desarrollar, que sirviese de herramienta de trabajo para los cuestionarios en las fases posteriores.

Con base en el proyecto Tuning, para mantenerlo dentro de las referencias del EEE, el equipo técnico definió las competencias genéricas 

FUNDACIÓN CAMPUS TECNOLÓGICO DE ALGECIRAS

como aquellas competencias transversales a la organización, asociadas a capacidades (nivel de desarrollo de aptitudes), que comprenden habilidades instrumentales (metodológicas, de procedimiento) de relación personal y sistémicas o integradoras.

Siguiendo las pautas anteriores, el equipo técnico limita un primer perfil de veinticuatro competencias genéricas que serán las propuestas para valoración en la primera oleada de cuestionarios enviados a los integrantes del panel o comités de expertos (tabla 2).

Tabla 2 Competencias Genéricas propuestas en la primera oleada
del Proyecto COGEMPLEO

\begin{tabular}{|c|c|c|}
\hline & DEFINICIÓN & COMPETENCIAS \\
\hline 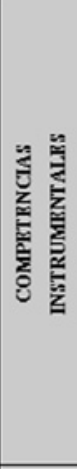 & $\begin{array}{l}\text { Competencias que tienen una función instrumental. } \\
\text { Entre ellas se incluyen: } \\
\text { - Habilidades cognoscitivas, la capacidad de } \\
\text { comprender y manipularideas y pensamientos. } \\
\text { - Capacidades metodológicas para manipular el } \\
\text { ambiente: ser capaz de organizar el tiempo y las } \\
\text { estrategias para el aprendizaje, tomar decisiones o } \\
\text { resolver problemas. } \\
\text { - Destrezas tecnológicas relacionadas con el uso } \\
\text { de maquinaria, destrezas de computación y } \\
\text { gerencia de la información. } \\
\text { - Destrezas lingüisticas tales como la } \\
\text { comunicación oral y escrita o conocimiento de } \\
\text { una segunda lengua. }\end{array}$ & $\begin{array}{l}\text { - Análisis y sintesis } \\
\text { - } \quad \text { Pranificación y Organización personal } \\
\text { - Comunicación oraly escrita } \\
\text { - } \text { lenocimiento de una segunda } \\
\text { - } \text { Manejo del ordenador } \\
\text { - Restión de la información } \\
\text { - Tomación de problemas } \\
\text { - Negociación } \\
\text { Persuasión e Influencia personal }\end{array}$ \\
\hline 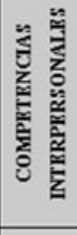 & $\begin{array}{l}\text { Habilidades individuales relativas a la capacidad de } \\
\text { expresar los propios sentimientos, habilidades criticas y } \\
\text { de autocrítica. } \\
\text { Destrezas sociales relacionadas con las habilidades } \\
\text { interpersonales, la capacidad de trabajar en equipo o la } \\
\text { expresión de compromiso social o ético. } \\
\text { Tienden a facilitar los procesos de interacción socialy } \\
\text { cooperación. }\end{array}$ & $\begin{array}{l}\text { - Capacidad crítica y autocrítica } \\
\text { - Habijo en equipo } \\
\text { - Compromiso ético }\end{array}$ \\
\hline 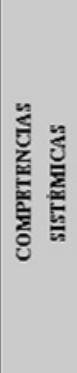 & $\begin{array}{l}\text { Son las destrezas y habilidades que conciemen a los } \\
\text { sistemas como totalidad. } \\
\text { Suponen una combinación de la comprensión, la } \\
\text { sensibilidad y el conocimiento que permiten al } \\
\text { individuo ver como las partes de un todo se relacionan } \\
\text { y se agupan. } \\
\text { Incluyen la habilidad de planificar los cambios de } \\
\text { manera que puedan realizarse mejoras en los sistemas } \\
\text { como un todo y diseñar nuevos sistemas. } \\
\text { Requieren como base la adquisición previa de } \\
\text { competencias instrumentales e interpersonales. }\end{array}$ & $\begin{array}{l}\text { - Orientación práctica } \\
\text { - Aprendizaje autónomo } \\
\text { - Liderazgo } \\
\text { - Desarrollo/apoyo de } \\
\text { colaboradores. } \\
\text { - Autonomia } \\
\text { - Iniciativa y espinitu } \\
\text { - Semprendedor } \\
\text { Sensibilidad a Costes, } \\
\text { - Ambiente, Calidady Medio } \\
\text { Motivación de logro }\end{array}$ \\
\hline
\end{tabular}

Fuente: FCTA: COGEMPLEO: Competencias Genéricas para el Empleo. Proyecto de Desarrollo de Competencias Genéricas para estudiantes de la Universidad de Cádiz (2009) 
MARÍA JOSÉ FONCUBIERTA RODRÍGUEZ, JOSÉ LUIS PEREA VICENTE Y GABRIEL GONZÁLEZ SILES

Determinación del panel de expertos

Para la constitución del panel de expertos, el equipo técnico decidió contar con cuatro subgrupos como colectivos de mayor interés y conocimiento sobre el objeto de estudio (tabla 3). Desde la perspectiva cualitativa, se buscaron personas bajo los criterios de experiencia, acceso a la información, posición, responsabilidad, disponibilidad y grado de compromiso. Desde el punto de vista cuantitativo, se procuró agilidad y eficiencia, a tenor del tiempo y los recursos disponibles.

Tabla 3

Comités de expertos para el Proyecto COGEMPLEO

\begin{tabular}{|c|c|}
\hline COMITÉS & INTEGRANTES \\
\hline $\begin{array}{l}\text { Representantes de } \\
\text { instituciones empresariales } \\
\text { y grandes empresas en la } \\
\text { comarca (AGI) }\end{array}$ & 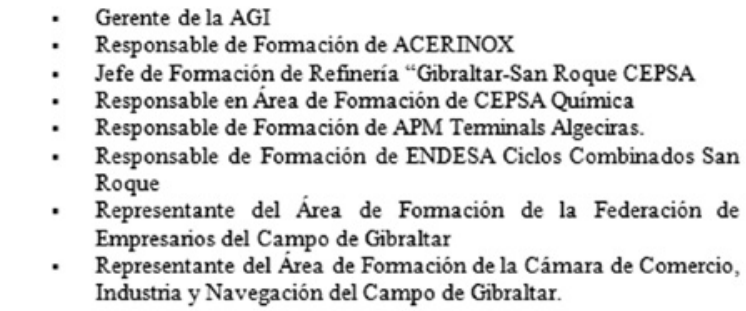 \\
\hline $\begin{array}{l}\text { Representantes de PYMES } \\
\text { de la Bahía de Algeciras y } \\
\text { Empresas Auxiliares de la } \\
\text { AGI con presencia en el } \\
\text { Campo de Gibraltar }\end{array}$ & $\begin{array}{ll}\text { - } & \text { Responsable de Formación de Eurogguas Algeciras S.A. } \\
\text { - } & \text { Responsable de Formación de INTECH (MAVISA) } \\
\text { - } & \text { Responsable de Formación de La Linea Vertical } \\
\text { - } & \text { Responsable de Formación de Preventor S.L. } \\
\text { - } & \text { Responsable de Formación de Diga } 33 \\
\text { - } & \text { Director Gerente de Incargo } \\
\text { - } & \text { Director de Cemaval } \\
\text { - } & \text { Responsable de RRHH de Cemaval } \\
\text { - } & \text { Gerente de Containers del Mediteráneo } \\
\text { - } & \text { Directora de Adecco Algeciras }\end{array}$ \\
\hline $\begin{array}{l}\text { Expertos en el ámbito de } \\
\text { las competencias para el } \\
\text { empleo en los colectivos } \\
\text { universitarios }\end{array}$ & $\begin{array}{l}\text { - Director General del Vicerrectorado Campus Bahia de Algeciras } \\
\text { - } \text { Profesor de Experto Universitario, y Jefe de Formación de } \\
\text { Refineria CEPSA } \\
\text { - Responsable de administración de la FCTA } \\
\text { - Técnico de Formación de la FCTA } \\
\text { - Coordinadora Cátedra Universitaria de ACERINOX } \\
\text { - Coordinador Cátedra Universitaria de CEPSA } \\
\text { - Coordinador de la Cátedra Universitaria de E-On } \\
\text { - Delegada del alumnado de la EPSA }\end{array}$ \\
\hline $\begin{array}{l}\text { Representante de } \\
\text { alumnado y recién } \\
\text { titulados EPSA }\end{array}$ & $\begin{array}{l}\text { Egresados de la EPSA en las titulaciones relacionadas conla Ingeniería en } \\
\text { Tecnologias Industriales: siete representantes del alumnado egresado }\end{array}$ \\
\hline
\end{tabular}

Fuente: Elaboración propia sobre información de FCTA (Documentos internos de trabajo, 2009)

Los criterios seguidos para la selección de los componentes de cada colectivo fueron:

- Instituciones empresariales y grandes empresas: se eligió a un representante de la Asociación de Grandes Industrias del Campo de 
Gibraltar (AGI) por su significativo peso en el empleo de la comarca (en torno al 33\% de la población ocupada, entre empleo directo, indirecto e inducido); a aquellas empresas que tienen Cátedras con la UCA o que han patrocinado o patrocinan cursos de postgrado en esta Universidad, y a miembros de las otras dos asociaciones empresariales más representativas del entorno: la Federación de Empresarios del campo de Gibraltar y la Cámara de Comercio.

- PYMES y empresas auxiliares: se seleccionaron empresas que habían colaborado previamente en acciones (cursos, conferencias, jornadas, etc.) para los universitarios, o a las que la FCTA había organizado proyectos formativos para sus plantillas.

- En el ámbito universitario: se formó el grupo con cargos académicos y con el profesorado coordinador de las distintas Cátedras de empresa, responsables de formación en la FCTA y la delegada del alumnado de la EPSA.

- En el colectivo de egresados: titulados que habían sido becarios, ayudantes de grupos de investigación en la EPSA o alumnos colaboradores y que se hubiesen incorporado al mercado laboral.

Primero en contacto telefónico, y posteriormente a través de correo electrónico, se informó a los seleccionados sobre los objetivos, la metodología, la duración estimada del estudio, así como de la difusión del Informe Final de resultados y sus potenciales aplicaciones.

\section{2. ${ }^{\circ} \quad$ Fase: rondas de cuestionarios al panel de expertos}

En una primera circulación, realizada en el mes de mayo de 2009, se solicitó a cada uno de los integrantes de los cuatro comités que, tras una reflexión personal, hicieran en el primer cuestionario (listado de competencias primero) una priorización de las competencias genéricas (de ocho a diez más importantes), valorándolas con: 1, a las de máxima importancia, y con 2 a las seleccionadas, pero de menor trascendencia que las anteriores. Se les dio, igualmente, la opción de indicar qué competencias, en su opinión, no deberían estar en ese listado, por no tener carácter genérico, y cuáles consideraban que faltaban.

Una vez recopilados los cuestionarios, y ante las ideas y opiniones reflejadas, el equipo técnico comenzó la segunda ronda de envíos del cuestionario, en el mes de junio de 2009, aportando, además, a los participantes, las reflexiones incorporadas en la primera circulación. Una de las más frecuentemente destacada fue la de añadir un criterio complementario, el de la viabilidad o facilidad de actuación formativa para la mejora de las capa- 
cidades asociadas a cada competencia. Si bien siempre es posible plantear actividades de información y concienciación, el perfil debía centrarse, aunque no limitarse, en aquellas competencias sobre las que más claramente se pudiera trabajar a nivel de actividad formativa. Con esta información se les pidió a los participantes que realizaran una nueva priorización.

Se evaluaron los resultados de esta segunda ronda, y, aunque en la concepción tradicional del método Delphi se exigen tres fases como mínimo (Linstone y Turoff, 1975), se siguió el actual modelo «Delphi modificado» (Moreno, 2002, Lee, 2009, Mengual, 2011), no siendo las diferencias sustanciales como para realizar una tercera, se dio por concluida la etapa de recogida de opinión de los expertos en cuanto al perfil de competencias buscado. Finalmente, se informó de los resultados a los participantes en el estudio.

\section{RESULTADOS}

De las dos fases de trabajo comentadas surgió el Perfil en Competencias Genéricas del Egresado, con las diez competencias que los expertos habían considerado como básicas, su priorización y el carácter otorgado a la misma, genérico o transversal (tabla 4). Se envió este documento a los participantes para su revisión y validación. Hemos de señalar que se dio un alto grado de consenso en cuanto a que competencias como las Habilidades Informáticas y de Gestión de la Información, aun siendo consideradas básicas para un titulado universitario, fueron descartadas del perfil final por tener un carácter más transversal que genérico por su componente «técnica» o instrumental implícita.

Una cuestión principal se ha de indicar, y es que cuando se realizó esta investigación (2009/2010) las titulaciones de Grados acababan de ponerse en marcha, con lo que el perfil del egresado sobre el que se trabajó pertenecía a un titulado en planes anteriores, es decir, en Ingenierías Técnicas de la EPSA. En sus asignaturas, por lo general, no se incluían competencias genéricas, o se incluían en escaso número. Estas han sido incorporadas en los nuevos títulos de Grado (en la EPSA: Ingeniería Civil e Ingeniería en Tecnologías Industriales: Mecánica, Electricidad, Electrónica y Química Industrial). No obstante, aunque, por este motivo, podría suponerse que las actuaciones formativas derivadas de este proyecto deberían ofrecerse a los estudiantes y egresados de los títulos a extinguir, teniendo en cuenta las competencias que se incluyen en las asignaturas de los Grados de EPSA, y siguiendo una de las conclusiones principales del estudio de la UNED que ya citamos en el primer apartado de este trabajo, sobre la dificultad de evaluar las competencias de carácter genérico, podemos concluir que también serán útiles al alumnado de dichos grados. 

FUNDACIÓN CAMPUS TECNOLÓGICO DE ALGECIRAS

Tabla 4

Perfil en Competencias Genéricas del Egresado

\begin{tabular}{|l|c|l|}
\hline \multicolumn{1}{|c|}{ Competencia } & Nivel de Prioridad & \multicolumn{1}{c|}{ Agrupación } \\
\hline Trabajo en equipo & 1 & Genérica \\
\hline Comunicación oraly escrita & 1 & Transversal \\
\hline Planificación y Organización & 1 & Genérica \\
\hline Resolución de problemas & 1 & Genérica \\
\hline Conocimiento de una segunda lengua & 1 & Transversal \\
\hline Toma de decisiones & 2 & Genérica \\
\hline Iniciativa y espíntu emprendedor & 2 & Genérica \\
\hline Autonomia & 2 & Genérica \\
\hline Aprendizaje autónomo & 2 & Genérica \\
\hline Innovación y Creatividad & 2 & Genérica \\
\hline
\end{tabular}

Fuente: FCTA. Proyecto COGEMPLEO. «Competencias Genéricas para el empleo». Informe Ejecutivo (junio, 2010)

Por otra parte, y en base a la unánime consideración por los participantes de la oportunidad y necesidad de una actuación como la que plantea el desarrollo de este proyecto, se les invitó, nuevamente, a razonar y comentar todos aquellos aspectos que considerasen importantes o interesantes con el fin de elaborar alternativas de actuación para la capacitación del alumnado en dichas competencias, objetivo final de este proyecto. Como resultado de este análisis, se elaboró una Propuesta Formativa a desarrollar por la FCTA, que recogiese acciones complementarias de formación para estudiantes y egresados.

Posteriormente, se presentó un Informe Final a los participantes en el estudio y a los agentes sociales y económicos del entorno, en el que figuraban tanto el Perfil como la Propuesta Formativa.

\section{ACTUACIONES FORMATIVAS DERIVADAS DEL ESTUDIO}

En el equipo del proyecto se debatieron y pusieron en común las propuestas realizadas por los expertos para trabajar las líneas de actuación derivadas de los resultados del estudio.

En primer lugar, se planteó el proyecto como un doble programa de desarrollo: por un lado será un apoyo para los estudiantes, que les permita complementar su perfil profesional a lo largo de su carrera, y, por otro, se entiende como una herramienta de actualización y «puesta en valor» para los titulados. Se decidió también que el proyecto contara con dos vías de participación:

- Una parte, a priori, abierta a todos los estudiantes y titulados de la EPSA, con actividades ofertadas desde el momento en que el 
MARÍA JOSÉ FONCUBIERTA RODRÍGUEZ, JOSÉ LUIS PEREA VICENTE Y GABRIEL GONZÁLEZ SILES

alumno acceda al Centro, y a posteriori, durante su incorporación al mundo profesional (Programa COGEMPLEO Abierto).

- Otro programa, cerrado a grupos preseleccionados, tanto de estudiantes como de titulados, a los que se les proporcione una formación más intensa y una acreditación (Programa "Diploma Cogempleo»). En la actuación formativa concreta se indicarán las condiciones o criterios a valorar para la selección de los grupos destinatarios.

En paralelo a este doble programa, se plantean otra serie de acciones complementarias (difusión, servicio de orientación, formación en competencias, integración en la metodología docente de las asignaturas y proyectos fin de título, apoyo al profesorado, colaboración con empresas mediante prácticas profesionales, etc.) que se detallan en las tablas 5 y 6.

Tabla 5

Líneas de Actuación propuestas en el Proyecto COGEMPLEO (Parte 1)

\begin{tabular}{|c|c|c|}
\hline ACTIVIDAD & MOMENTO & METODOLOGIA Y MEDIOS \\
\hline $\begin{array}{l}\text { 1. Difusión del Proyecto y del Modelo } \\
\text { de Competencias Genéricas (C.G.), } \\
\text { información sobre medios y actividades } \\
\text { a disposición de alumnado y titulados. } \\
\text { Promoción del desanrollo personal como } \\
\text { complemento a las asignaturas. }\end{array}$ & $\begin{array}{l}\text { Desde el ingreso en el } \\
\text { Centro. } \\
\text { Intensivo a principios } \\
\text { de cada curso. } \\
\text { Alcanza a recién } \\
\text { titulados. }\end{array}$ & $\begin{array}{l}\text { Presentaciones/Conferencias. } \\
\text { Acciones de difusión/ publicidad. } \\
\text { Apoyo informático on-line. } \\
\text { Consolidación de un Equipo de } \\
\text { Proyecto para liderarlo. }\end{array}$ \\
\hline $\begin{array}{l}\text { 2. Asesoramiento personalizado para } \\
\text { identificación de necesidades } \\
\text { individuales y confección de itinerarios } \\
\text { de desarrollo de C.G. }\end{array}$ & $\begin{array}{l}\text { A lo largo de toda la } \\
\text { carrera y titulados. } \\
\text { Intensivo a principios } \\
\text { de cada curso. }\end{array}$ & $\begin{array}{l}\text { Técnicos de Desarrollo } \\
\text { asesoren pue } \\
\text { alumnado y profonalmente } \\
\text { Espacio fisico y material de apoyo. }\end{array}$ \\
\hline $\begin{array}{l}\text { 3. Programa Base COGEMPLEO de } \\
\text { actividades de sensibilización para } \\
\text { desarrollar competencias sistemáticas } \\
\text { (innovación, autonomia, creatividad, } \\
\text { aprendizaje autónomo). }\end{array}$ & $\begin{array}{l}\text { Durante la carrera y a } \\
\text { posterion. } \\
\text { Programa anual de } \\
\text { actividades. }\end{array}$ & $\begin{array}{l}\text { Seminarios, conferencias de } \\
\text { expertos, talleres. } \\
\text { Profesionales, expertos, monitores } \\
\text { especializados. } \\
\text { Libre acceso previa inscripción. } \\
\text { Posibilidad de certificación. }\end{array}$ \\
\hline $\begin{array}{l}\text { 4. Oferta de Acciones de Desarrollo de } \\
\text { competencias transversales: formación } \\
\text { gratuita en el grupo de competencias } \\
\text { identificadas como transversales: cursos } \\
\text { especificos de cualificación } \\
\text { (herramientas informáticas, inglés, } \\
\text { exposición, redacción,...). }\end{array}$ & $\begin{array}{l}\text { Durante la carrera y a } \\
\text { posterion. } \\
\text { Intensivo en momentos } \\
\text { de baja actividad } \\
\text { académica. } \\
\text { En continuo a titulados. }\end{array}$ & $\begin{array}{l}\text { Oferta de cursos intensivos de corta } \\
\text { duración para capacitación a todos } \\
\text { los niveles. }\end{array}$ \\
\hline $\begin{array}{l}\text { 5. Formación y apoyo a profesorado en } \\
\text { C.G.: coordinación de metodologias y } \\
\text { criterios de evaluación. }\end{array}$ & $\begin{array}{l}\text { Intensivo antes del } \\
\text { comienzo de curso o } \\
\text { cuatrimestre } \\
\text { Continúo durante el } \\
\text { curso. }\end{array}$ & $\begin{array}{l}\text { Combinación de formación y } \\
\text { orientación por Técnicos de } \\
\text { Desarrollo, tutonzado por un } \\
\text { facilitador/director. Trabajo gnupal } \\
\text { (Comunidad de proyecto). } \\
\text { Creación de un Vivero de } \\
\text { innovación pedagógica. }\end{array}$ \\
\hline $\begin{array}{l}\text { 6. Integración del desarrollo de C.G. en } \\
\text { asignaturas de últimos cursos. }\end{array}$ & $\begin{array}{l}\text { Durante el último } \\
\text { penúltimo curso. }\end{array}$ & $\begin{array}{l}\text { Aplicación de metodologias } \\
\text { previstas y de sistema de } \\
\text { evaluación homogeneizado. }\end{array}$ \\
\hline
\end{tabular}

Fuente: FCTA. Proyecto COGEMPLEO. «Competencias Genéricas para el empleo». Informe Ejecutivo (junio, 2010) 

FUNDACIÓN CAMPUS TECNOLÓGICO DE ALGECIRAS

Tabla 6

Lineas de Actuación propuestas en el Proyecto COGEMPLEO (Parte 2)

\begin{tabular}{|c|c|c|}
\hline $\begin{array}{l}7 . \quad \text { Programa Cualificación } \\
\text { COGEMPLEO de formación especifica } \\
\text { en C. G. (trabajo en equipo, toma de } \\
\text { decisiones, planificación y organización, } \\
\text { resolución de problemas) para alumnado } \\
\text { próximo a egresar y titulados, con } \\
\text { acceso voluntario según un baremo de } \\
\text { evaluación. Programa completo con } \\
\text { Diploma final. }\end{array}$ & $\begin{array}{l}\text { Actuaciones intensivas } \\
\text { al inicio y al final del } \\
\text { curso. } \\
\text { Programa anual para el } \\
\text { alumnado en su } \\
\text { penúltimo ó último año } \\
\text { de estudios, así como } \\
\text { recién titulados. }\end{array}$ & $\begin{array}{l}\text { Cursos específicos en C.G. } \\
\text { Formación durante el curso } \\
\text { académico. } \\
\text { Criterios de acceso valorando } \\
\text { aspectos académicos y de } \\
\text { capacitación previa. }\end{array}$ \\
\hline $\begin{array}{l}\text { 8. Programa Proyecto fin de carrera } \\
\text { COGEMPLEO consistente en la } \\
\text { selección de un grupo de Diplomados } \\
\text { COGEMPLEO para la realización del } \\
\text { Proyecto Fin de Carrera (PFC), con } \\
\text { apoyo continuo tanto técnico como de } \\
\text { desarrollo de C.G. }\end{array}$ & $\begin{array}{l}\text { Al final de la carrera. } \\
\text { Programa intensivo } \\
\text { sontinue durante el } \\
\text { desarrollo del PFC. }\end{array}$ & $\begin{array}{l}\text { Dedicación de profesorado para la } \\
\text { tutoria continua del proyecto. } \\
\text { Selección de proyectos especificos } \\
\text { para el Programa COGEMPLEO. } \\
\text { Ampliación del ámbito habitual del } \\
\text { PFC a aspectos empresariales. }\end{array}$ \\
\hline $\begin{array}{l}\text { C. Creación la Biblioteca } \\
\text { COGEMPLEO, dotada de recursos } \\
\text { fisicos y virtuales (on-line) que permita } \\
\text { la autogestión de perfiles y el } \\
\text { autodesarrollo al alumnado interesado. }\end{array}$ & Servicio continuo. & $\begin{array}{l}\text { Portal web y espacio físico para } \\
\text { consulta. } \\
\text { Mantenimiento y actualizacion } \\
\text { continua de materiales e } \\
\text { informaciones. }\end{array}$ \\
\hline $\begin{array}{l}\text { 10. Establecimiento de la figura del } \\
\text { Colaborador COGEMPLEO: egresados, } \\
\text { alumnado de otros centros, expertos } \\
\text { extemos, etc., que participen } \\
\text { voluntariamente en el programa, } \\
\text { apoyando actividades del proyecto, a la } \\
\text { par que reciben apoyo y formación para } \\
\text { su propio desarrollo. }\end{array}$ & $\begin{array}{l}\text { Continuo, en función } \\
\text { de la organización de } \\
\text { cada actividad y etapa. } \\
\text { Especial atención a los } \\
\text { reciên titulados de } \\
\text { EPSA (1 o } 2 \text { años). }\end{array}$ & $\begin{array}{l}\text { Participación bajo supervisión de } \\
\text { los Técnicos de Desarrollo } \\
\text { apoyando al alumnado en áreas } \\
\text { concretas. } \\
\text { Participación en actividades de los } \\
\text { programas formativos, con } \\
\text { certificación. }\end{array}$ \\
\hline $\begin{array}{l}\text { 11. Valoración de la viabilidad y, en su } \\
\text { caso, diseño de un programa especifico } \\
\text { de Prácticas profesionales } \\
\text { COGEMPLEO que apoyen el Desanrollo } \\
\text { de C.G., asi como de una Bolsa de } \\
\text { Trabajo para estudiantes y egresados. }\end{array}$ & $\begin{array}{l}\text { Al final del curso } \\
\text { académico. }\end{array}$ & $\begin{array}{l}\text { Valorar viabilidad y, en su caso, } \\
\text { definir detalles con colaboración de } \\
\text { representantes de empresas. }\end{array}$ \\
\hline
\end{tabular}

Fuente: FCTA. Proyecto COGEMPLEO. «Competencias Genéricas para el empleo». Informe Ejecutivo (junio, 2010)

Al respecto, las primeras acciones desarrolladas se refieren a:

- La línea de actuación 1, por medio de un proceso de difusión, tanto en la propia EPSA, como en las instituciones empresariales de la comarca, y entre los medios de comunicación del entorno.

- Una combinación de las líneas 3, 4 y 8, sobre formación en las competencias señaladas en el perfil, tanto a estudiantes de los últimos cursos como a egresados de las titulaciones de la EPSA relacionadas con las Ingenierías en Tecnologías Industriales (como colectivos destinatarios preferentes), a través de un Experto Universitario en Competencias Genéricas para el Empleo.

- Una acción que redefine y engloba las líneas 9 y 11, consistente en un modelo integral, de marco virtual, que enlace las ofertas de los 
MARÍA JOSÉ FONCUBIERTA RODRÍGUEZ, JOSÉ LUIS PEREA VICENTE Y GABRIEL GONZÁLEZ SILES

alumnos o egresados que deseen encontrar empleo en el entorno empresarial (mediante breves cartas de presentación del perfil adquirido) y las demandas de dichas empresas (a través de un breve modelo de competencias o perfil deseado); un análisis periódico, y, por tanto, dinámico, de tales necesidades empresariales, con las que actualizar y complementar el Proyecto COGEMPLEO; y, en consecuencia, la determinación de un programa de acciones formativas pertinentes.

\section{REFLEXIONES FINALES}

El Proyecto cumple con el espíritu de múltiples estudios y reflexiones cuando indica que la empleabilidad ha de surgir de la diversidad de acciones formativas que, entre otras materias, capaciten en competencias genéricas, y que este ha de ser uno de los puntos de análisis en la creación de programas y unidades de formación. Lo hace, además, desde una perspectiva eminentemente práctica, fruto de una estrecha colaboración y relación Universidad/entorno socioempresarial en la comarca del Campo de Gibraltar, siguiendo una filosofía y un procedimiento que bien pueden servir de modelo para experiencias similares en otras zonas o entornos.

Lo peculiar de este proyecto son la participación y el apoyo directos de las empresas que conforman el tejido empresarial del entorno. Esto es consecuencia de la estrecha relación de las empresas de la Bahía, sobre todo de la gran industria, con la Universidad. Fruto de ello, son las tres Cátedras de Empresa-UCA que existen en la comarca: Cátedras CEPSA, ACERINOX y E-On (antigua ENDESA). Esto permite una comunicación muy fluida entre las partes, y como consecuencia, un conocimiento casi a tiempo real de las necesidades de las empresas. De hecho, son las empresas de la AGI quienes demandan y solicitan a las distintas instituciones unos recursos humanos más y mejor formados en competencias de carácter genérico, necesarias para el normal desarrollo de sus obligaciones y responsabilidades diarias en su puesto de trabajo. Y es a partir del momento en el que la Universidad y la FCTA son conocedoras de estas demandas, cuando ponen en marcha los mecanismos necesarios para darle respuesta.

Representa el tipo de paso firme que se debe dar para conseguir paliar uno de los problemas que viene encontrándose el tejido empresarial: titulados con una carga importante de conocimientos, pero una significativa deficiencia en competencias necesarias para enfrentarse al mundo laboral. Conscientes de que el paso de la Universidad al mundo laboral es uno de los tránsitos más complicados en la vida de una persona, con este tipo de proyectos pretendemos conseguir que sea lo menos traumático posible. Aún así, 
existen competencias y enseñanzas que solo la experiencia y el día a día en el trabajo pueden otorgar, pero colaboraciones como la que hemos presentado en este trabajo pueden servir de catalizador a tal fin.

Mediante una metodología participativa, de consulta y consenso con expertos, este Proyecto ha demostrado que las empresas y demás entidades demandan un desarrollo integral y completo de la persona, en todo su abanico de capacidades. La valoración de las competencias genéricas es un factor determinante en los procesos de selección por parte de estas. Por ello, un universitario que complemente su currículo académico con formación reconocida en este tipo de capacidades incrementa sus posibilidades de acceder al mundo laboral, mejora su desempeño y, por tanto, su valor para la empresa, su empleabilidad.

La idea que subyace en este planteamiento es la que expone Rodríguez Moreno (2006) con el denominado «balance de competencias», o comparación entre las competencias que un individuo posee y las que necesita poseer para desarrollar un perfil profesional que le suministre empleabilidad, en este caso, según las demandas del entorno más cercano. El balance de competencias hace reflexionar a la persona, en este caso al estudiante o egresado universitario, sobre los pasos o actividades de capacitación a dar en su proyecto profesional.

Como paso lógico, el proyecto termina pasando de la investigación a la acción, desarrollando una serie de acciones que persiguen complementar la cualificación de los estudiantes y recién titulados de la EPSA en dichas competencias. Como fruto de la activación de estos mecanismos se ha creado en el Campus Bahía de Algeciras, de la mano de la UCA y de la FCTA, el Titulo Experto Universitario en Competencias y Habilidades para el Desarrollo Profesional. Agrupa las diez competencias del perfil determinado en el análisis de expertos comentado en este trabajo. Su primera edición se está llevando a cabo en el presente curso 2013/14. Complementa, por tanto, la formación ya adquirida por los egresados, para su adecuación al mundo laboral. Consideramos de gran interés el análisis de esta primera edición, y, por tanto, podría ser tema para un nuevo trabajo, donde se exponga el origen de la idea, su creación, el desarrollo, la participación de las empresas en su impartición, la metodología didáctica, los resultados, y el feedback de las empresas.

Por otra parte, el propio desarrollo por parte de la FCTA de las actuaciones a poner en marcha en cumplimiento de la propuesta formativa derivada del Proyecto COGEMPLEO, así como la continuidad del compromiso de las empresas del entorno con ellas consituyen, por sí mismas, futuras líneas de investigación. 


\section{NOTAS}

1. La comarca del Campo de Gibraltar está constituida por siete municipios: uno en su vertiente atlántica, Tarifa, dos en el interior: Castellar de la Frontera y Jimena de la Frontera, y cuatro en lo que se denomina propiamente Bahía de Algeciras, en su vertiente mediterránea: Algeciras, Los Barrios, San Roque y La Línea de la Concepción.

2. CHEERS y REFLEX, han proporcionado información sobre la relación entre la educación superior recibida y la empleabilidad de los egresados, unos años después de adquirir estos su titulación. Para mayor información puede consultarse: Allen, J., Van der Velden, R. (eds.) (2007): The Flexible Professional in the Knowledge Society. General Results of the REFLEX Project. Maastricht: Research Centre for Education and the Labour Market; y Schomburg, H., Teichler, U. (2006): Higher Education and Graduate Employment in Europe. Results from Graduate Surveys from Twelve Countries. Dordrecht: Springer.

3. UEConverge. Fundación Universidad Empresa de la Cámara de Comercio de Madrid. http:// www.fue.es/HTML/071_ueconverge.asp

4. Proyecto DeSeCo (Definition and Selection of Competencies) OCDE. Recuperado de http://www. oecd.org/education/skills-beyond-school/definitionandselectionofcompetenciesdeseco.htm

5. University of Cambridge. Transferable Skills Website. Transferable Skills for Undergraduate Students. Recuperado de http://www.skills.cam.ac.uk/

6. En este proyecto la Universidad de Surrey, junto con otras del Reino Unido, identifican tres modelos de intervención en las competencias genéricas: inducido (desarrollar las habilidades); integrado (las habilidades se enseñan y practican dentro de los créditos normales de formación); y específico (configuración de módulos específicos que permiten el desarrollo de competencias).

7. FGULEM. http://www.fgulem.es/certingles/certicap.html 


\section{REFERENCIAS BIBLIOGRÁFICAS}

ANECA (s.f.). Programa VERIFICA. Recuperado de http://www.aneca.es/Programas/VERIFICA

Ansorena Cao, A. (1996). Quince pasos para la selección de personal con éxito. Madrid: Paidós.

Bennett, N., Dunne, E. \& Carré, C. (1999). Patterns of core and generic skill provision in higher education. Higher Education, 37, 71-93

Blanco, A. (coord.) (2009). Desarrollo y evaluación de competencias en Educación Superior. Madrid: Narcea.

Calderón, C. y Escalera Izquierdo, G. (2008). La evaluación de la docencia ante el reto del Espacio Europeo de Educación Superior (EEES). Educación XX1, 11, 237-256.

Corominas, E., Tesouro, M., Capell, D., Teixidó, J., Pèlach, J. y Cortada, R. (2006). Percepciones del profesorado ante la incorporación de las competencias genéricas en la formación universitaria. Revista de Educación, 341, 301-336.

Corominas, E. (2001). Competencias genéricas en la formación universitaria. Revista de Educación, 325, 299-321.

Declaración de Bolonia (1999). El espacio europeo de la enseñanza superior. Bolonia, 19 de Junio de 1999. Recuperado de http://www.eees.es/pdf/Bolonia_ES.pdf

Fallows, S. \& Steven, C. (2000). The skills agenda, in Fallows, S. and Steven, C. Integrating key skills in higher education; employability, transferable skills and learning for life. London: Kogan Page.

González, J. y Wagenaar, R. (eds.) (2003). TUNING Educational Structures in Eu- rope. Informe Final. Fase Uno. Bilbao: Universidad de Deusto/ Universidad de Groningen.

Gutiérrez, S. y De Pablos, C. (2010). Análisis y evaluación de la gestión por competencias en el ámbito empresarial y su aplicación a la Universidad. Revista Complutense de Educación, 21(2), 323-343.

Heijke, H., Meng, C. \& Ris, C. (2003). Fitting to the Job: the role of the generic and vocational competencies in Adjustment and Performance. Labour Economics, 10(2), 215-229.

IUED. Instituto Universitario de Educación a Distancia de la UNED. (2010). Del diseño a la evaluación de competencias genéricas: análisis empírico e intervención mediante rúbricas. Proyecto EA 2009-2012. Madrid: UNED.

Jornet Meliá, J. M., González Such, J., Suárez Rodríguez, J. M. y Perales Montolío, M. J. (2011). Diseño de procesos de evaluación de competencias: consideraciones acerca de los estándares en el dominio de las competencias. Bordón. Revista de Pedagogía, 63(1), 125-145.

Lee, Y. (2009). Competencias Hended by Korean HRD Master's Graduates: A Compararison between the ASTD WLP Competency Model and the Korean Study. Human Resource Development Quarterly, 20(1), 107-133.

Ley Orgánica 4/2007, de 12 de abril, por la que se modifica la Ley Orgánica 6/2001, de 21 de diciembre, de Universidades

Linstone, H. A., \& Turoff, M. (1975). The Delphi method: Techniques and applications. Reading, MA.: Addison WEsley Publishing. 
Martín del Peso, M., Rabadán Gómez, A. B. y Hernández March, J. (2013). Desajustes entre formación y empleo en el ámbito de las enseñanzas técnicas universitarias: la visión de los empleadores de la Comunidad de Madrid. Revista de la Educación, 360, 244-267.

Mengual, S. (2011). La importancia percibida por el profesorado y el alumnado sobre la inclusión de la competencia digital en educación Superior. Alicante: Departamento de Didáctica General y Didácticas específicas. Facultad de Alicante.

Ministerio de Educación (2013). Marco Español de Cualificaciones para la Educación Superior (MECES). Recuperado de enhttp://www.mecd.gob.es/cniie/ investigacion-innovacion/marcos-cualificaciones/meces.html

Moreno, E. et al. (2002). La técnica «Delphi» en la evaluación de necesidades: una aplicación al tratamiento del género en los centros escolares. Bordón. Revista de Pedagogía, 54(1), 83-94

OCDE (2008). Education at a glance. Paris: OCDE.

Real Decreto 1393/2007, de 29 de octubre, que establece la ordenación de las enseñanzas universitarias oficiales.

Real Decreto 861/2010, de 2 de julio, por el que se modifica el Real Decreto 1393/2007, de 29 de octubre.

Ribes Iñesta, E. (2011). El concepto de competencia: Su pertinencia en el de- sarrollo psicológico y la educación. Bordón. Revista de Pedagogía, 63(1), 33-45.

Rodríguez Moreno, M. L. (2006). Evaluación, Balance y Formación de competencias laborales transversales. Propuesta para mejorar la calidad en la formación profesional y en el mundo del trabajo. Barcelona: Laertes.

Tejada, J. y Navío, A. (2005). El desarrollo y la gestión de competencias profesionales: una mirada desde la formación. Revista Iberoamericana de Educación (versión digital), 37(2). Recuperado de http://www.rieoei.org/ deloslectores/1089Tejada.pdf

Universidad de Sevilla (s.f.). Descriptores de Dublín. Informe en español. Recuperado de

http://institucional.us.es/eees/formacion/ descriptores_Dublin_castellano.pdf

Van der Hofstadt, C. y Gómez Gras, J. M. (coords.) (2006). Competencias y habilidades profesionales para universitarios. Madrid: Díaz de Santos.

Vélaz de Medrano, C. (2008). Formación y Profesionalización de los Orientadores desde el Enfoque de Competencias. Educación XX1, 11, 155-181.

Villa Sánchez, A. y Poblete Ruiz, M. (2011). Evaluación de competencias genéricas: Principios, oportunidades y limitaciones. Bordón. Revista de Pedagogía, 63(1), 147-170. 


\section{PERFIL ACADÉMICO Y PROFESIONAL DE LOS AUTORES}

María José Foncubierta Rodríguez, Profesora del área de Organización de Empresas en la Universidad de Cádiz. Directora de la Facultad de Ciencias Económicas y Empresariales en su sede del Campus Bahía de Algeciras. Doctora por la UNED, compagina su docencia y gestión con la investigación en formación profesional continua y estrategias de recursos humanos en grupos de investigación, nacionales e internacionales. Ha participado en proyectos de adaptación al EEES en el Campus Bahía de Algeciras.

José Luis Perea Vicente, Doctorando de la Universidad de Cádiz, en investigación sobre formación y evaluación del personal. Licenciado en Administración y Dirección de Empresas por la UCA. Desde que finalizó su carrera ha estado ligado a la empresa privada, trabajando en puestos de dirección y gestión en el departamento de Recursos Humanos durante cuatro años. Actualmente trabaja como Asesor Económico Personal Objetivo, en una Consultoría de Finanzas.

Gabriel González Siles, Profesor del área de Máquinas y Motores Térmicos en la Universidad de Cádiz. Director de la Escuela Politécnica Superior de Algeciras. Forma parte del grupo de investigación «Grupo de Ingeniería Térmica (TEP221)», cuyas líneas de investigación están relacionadas con la energía en la edificación, los sistemas de climatización y la integración de las energías renovables en los edificios. Ha participado en proyectos de calidad, excelencia y evaluación de competencias y formación continua.

Dirección de los Autores: María José Foncubierta Rodríguez

Escuela Politécnica Superior de Algeciras (EPSA)

Avda. Ramón Puyol, s/n

11202 - Algeciras (Cádiz)

E-mail: mariajose.foncubierta@uca.es

José Luis Perea Vicente

Consultoría de Finanzas

Urb. El Bosque, C/ Ursulinas bl.4 Locales

11 y 12

11405 Jerez de la Frontera

E-mail: jluis.pereav@gmail.com

Gabriel González Siles

Escuela Politécnica Superior de Algeciras (EPSA) 
MARÍA JOSÉ FONCUBIERTA RODRÍGUEZ, JOSÉ LUIS PEREA VICENTE Y GABRIEL GONZÁLEZ SILES

$$
\begin{aligned}
& \text { Avda. Ramón Puyol, s/n } \\
& 11202 \text { - Algeciras (Cádiz) } \\
& \text { E-mail: gabriel.gonzalez@uca.es }
\end{aligned}
$$

Fecha Recepción del Artículo: 22. Abril. 2013

Fecha modificación Artículo: 10. Diciembre. 2013

Fecha Aceptación del Artículo: 17. Marzo. 2014

Fecha Revisión para Publicación: 05. Julio. 2015 
